\title{
Vitamina D y enfermedades respiratorias pediátricas
}

\section{Vitamin $\mathrm{D}$ and pediatrics respiratory diseases}

\author{
Javier Cepeda S. ${ }^{a}$, Daniel Zenteno A. ${ }^{a}$, Claudia Fuentes S. ${ }^{b}$, Raúl Bustos B. ${ }^{c}$
}

aServicio de Pediatría, Hospital Clínico Regional Guillermo Grant Benavente. Concepción, Chile bDepartamento de Pediatría, Facultad de Medicina, Universidad de Concepción. Concepción, Chile

'Medicina Intensiva Pediátrica, Clínica Sanatorio Alemán y Hospital Guillermo Grant Benavente. Concepción, Chile

Recibido el 18 de mayo de 2018; aceptado el 11 de octubre de 2018

\section{Resumen}

El mejor entendimiento sobre la actividad global de la vitamina D, ha llevado a una intensa búsqueda de sus implicancias en enfermedades no esqueléticas. En este artículo se presenta una revisión actualizada de la relación entre la vitamina D y la patología respiratoria pediátrica. Se realizó una búsqueda bibliográfica en PUBMED utilizando términos libres y MESH: vitamina D, enfermedades del sistema respiratorio, asma, bronquiolitis. Se seleccionó estudios en humanos menores de 18 años y animales, publicados en inglés y español hasta el 2017. Se encontraron 507 artículos, de los cuales se incluyeron 43. Evidencia indirecta apunta hacia un rol de la vitamina D y la maduración pulmonar fetal. En relación a la patología pulmonar pediátrica, los estudios son escasos y poco concluyentes. Nuevos meta - análisis, con evaluación individualizada de los participantes, muestran un importante rol protector de la suplementación en la prevención de exacerbaciones asmáticas severas e infecciones virales agudas. En bronquiolitis los resultados son contradictorios, sin relación clara entre niveles plasmáticos y severidad. No existe suficiente evidencia que evalué los beneficios en fibrosis quística y tuberculosis. Recientemente se ha propuesto una relación directa entre la severidad de los trastornos respiratorios del sueño y los niveles plasmáticos de vitamina $\mathrm{D}$, aunque se desconoce los mecanismos exactos involucrados a esta asociación. La información actual permite suponer que la suplementación de vitamina D puede representar una estrategia costo - efectiva en la reducción de importantes causas de morbimortalidad infantil.

\section{Abstract}

The better understanding of the global activity of vitamin D has led to an intense search for its involvement in non-skeletal diseases. This article presents an updated review of the relationship between vitamin D and pediatric respiratory pathology. A literature search was performed in PUBMED using free terms and MESH terms: vitamin D, asthma, respiratory system diseases, and bronchiolitis. Studies in human patients younger than 18 years and animals, published in English and Spanish until
Palabras clave:

Vitamina D;

Enfermedad del

Sistema Respiratorio;

Asma;

Niño;

Bronquiolitis

Keywords:

Vitamin D;

Respiratory System

Disease;

Asthma;

Child;

Bronchiolitis 
2017 were included. 507 articles were found, of which 43 were included. Indirect evidence suggests a role of vitamin $\mathrm{D}$ and fetal lung maturation. In relation to pediatric pulmonary pathology, studies are scarce and inconclusive. Recent meta-analyses performed with individualized evaluation of the participants shows an important protective role of vitamin D supplementation in the prevention of severe asthma exacerbations and acute viral infections. In bronchiolitis, the results are contradictory, with no clear relationship between plasma levels and severity. There is not enough evidence to assess the benefits of vitamin D supplementation in cystic fibrosis and tuberculosis. A direct relationship between the severity of sleep-related breathing disorders and vitamin D plasma levels has recently been proposed, although the exact mechanisms involved in this association are unknown. Current information suggests that vitamin D supplementation may represent a cost-effective strategy in reducing important causes of infant morbidity and mortality.

\section{Introducción}

El interés en la vitamina D se ha reavivado sustancialmente durante la década pasada. El descubrimiento de su eje endocrino y funcionamiento a través de un receptor nuclear ubicado en tejidos no relacionados con el calcio o hueso ha llevado a la investigación de su acción en patologías no esqueléticas ${ }^{1}$. A la fecha, se le ha implicado en la patogenia de enfermedades autoinmunes, cardiovasculares y pulmonares como la enfermedad pulmonar obstructiva crónica (EPOC) y el asma ${ }^{2,3}$. La mayoría de los estudios se ha enfocado en los mecanismos inmunes de la enfermedad pulmonar, no obstante, el impacto de la vitamina $\mathrm{D}$ en el desarrollo precoz y maduración pulmonar es un campo emergente de investigación ${ }^{4,5}$. En este sentido, la hipovitaminosis $\mathrm{D}$ durante el embarazo, se ha relacionado con un menor desarrollo placentario, parto prematuro, síndrome de distress respiratorio (SDR) y displasia broncopulmonar $(\mathrm{DBP})^{6}$. Aún más, estudios sugieren un rol en la embriogénesis y diferenciación celular pulmonar, en la que su deficiencia podría agravar la enfermedad pulmonar del prematuro ${ }^{7}$.

En nuestro país, Le Roy y cols. evaluaron los niveles plasmáticos de vitamina $\mathrm{D}$ en 60 preescolares sanos provenientes de zonas australes, presentando un $64 \%$ valores en rango de deficiencia $(<20 \mathrm{ng} / \mathrm{mL})^{8}$.

El objetivo de la presente revisión es realizar una revisión bibliográfica actualizada sobre vitamina $\mathrm{D}$ y su asociación con el desarrollo pulmonar y la patología respiratoria, específicamente en asma y atopía, fibrosis quística, infecciones respiratorias y trastornos respiratorios del sueño (TRS). No se realizó una revisión sistemática del tema a tratar.

\section{Metodología}

Se realizó una búsqueda bibliográfica en PUBMED combinando los términos libres y MESH: "Vitamin D", "Respiratory Tract Diseases", "Respiratory Tract Infections", "Asthma”, "Cystic Fibrosis", "Sleep Apnea", "Allergy and Inmunology", "Lung”, "Bronchioli- tis” AND “Child. Se incluyeron artículos publicados en inglés y español hasta el año 2017, tanto en humanos como animales y limitados a menores de 18 años. En base a esto se redujo el total inicial de 507 artículos a 43, excluyendo además aquellos en que no fue posible acceder al artículo completo o no existió relación entre el articulo y la revisión. Adicionalmente, se amplió la búsqueda a las referencias de los trabajos seleccionados. Los artículos seleccionados correspondieron a revisiones bibliográficas, estudios clínicos y revisiones sistemáticas. Este manuscrito no pretende ser una revisión sistemática de la evidencia.

\section{Metabolismo de la vitamina D}

Los humanos obtienen la vitamina $\mathrm{D}$ como consecuencia de su exposición a la luz solar (principal determinante), a través de la dieta (Vitamina D3 o Colefalciferol en productos de origen animal y Vitamina D2 o Ergocalciferol de origen vegetal) y la suplementación ${ }^{9}$.

La radiación solar ultravioleta $B$ (longitud de onda entre 290 a $315 \mathrm{~nm}$ ), penetra en la piel y convierte el 7-dehidrocolesterol a previtamina $\mathrm{D} 3$, la que rápidamente isomeriza y se transforma en vitamina D3, en un proceso dependiente de calor. La vitamina D3 en sangre se une a su proteína transportadora y es llevada al hígado donde es metabolizada por la 25 - Hidroxilasa a 25 - Hidroxivitamina D (25 (OH) D). Esta, a su vez, es modificada a nivel renal por la enzima 25-hidroxivitamina D-1 $\alpha$ hidroxilasa (CYP27B1) a su forma activa, la 1,25-dihidroxivitamina $\mathrm{D}\left(1,25(\mathrm{OH})_{2} \mathrm{D}\right)^{10}$.

La producción renal de $1,25(\mathrm{OH})_{2}$ D está estrechamente regulada por los niveles plasmáticos de la hormona paratiroidea (PTH), calcio y fosforo. La 1,25 $(\mathrm{OH})_{2} \mathrm{D}$ disminuye su propia síntesis a través de un feedback negativo, así como la síntesis y secreción de PTH por la glándula paratiroides ${ }^{10,11}$. A su vez, la 1,25 $(\mathrm{OH})_{2} \mathrm{D}$ incrementa la absorción en el intestinal delgado de calcio. En los osteoblastos, induce la maduración del pro-osteoclasto a osteoclastos maduros, los que remueven calcio y fosforo desde el hueso, manteniendo sus niveles plasmáticos en sangre ${ }^{11}$. 


\section{Maduración pulmonar}

La actividad de la $1,25(\mathrm{OH})_{2}$ D se encuentra mediada por su unión al receptor nuclear (VDR), un miembro de la superfamilia de los receptores de esteroides $^{12}$. El VDR es un factor de transcripción ligandodependiente que altera la expresión de ciertos genes en respuesta a la 1,25 $(\mathrm{OH})_{2} \mathrm{D}$. El complejo VRD-Ligando forma un heterodímero con el receptor de retinoides $X(R X R)$ y se une a elementos sensibles a vitamina D (VDREs) localizados en las regiones promotoras de genes diana'. Estos complejos pueden promover o suprimir la transcripción de un amplio rango de genes. Estudios en ratas knock-out para VDR han evidenciado el desarrollo precoz de enfisema, declinación de la función pulmonar y cambios inflamatorios a nivel pulmonar ${ }^{13}$. No obstante, estudios de los efectos de las variaciones del material genético en la síntesis, transporte, degradación y acción de la vitamina $\mathrm{D}$, como consecuencia de polimorfismos o la ausencia del gen en el desarrollo pulmonar temprano, son todavía es$\operatorname{casos}^{14}$.

Durante el embarazo, los niveles plasmáticos maternos de $25(\mathrm{OH})$ D se mantienen sin cambios, a diferencia de la 1,25 $(\mathrm{OH})_{2}$ que incrementa varias veces su valor desde etapas tempranas y se mantiene alto durante todo el embarazo ${ }^{15}$. El feto no posee producción endógena de $25(\mathrm{OH}) \mathrm{D}$, siendo totalmente dependiente de la transferencia materna. La hipovitaminosis $\mathrm{D}$ es frecuente durante el embarazo y en los neonatos, especialmente aquellos prematuros, debido a que la transferencia placentaria de $25(\mathrm{OH})$ D ocurre principalmente durante el último trimestre ${ }^{12}$. En el año 1999, Blackstrom y cols., randomizaron 39 prematuros de menos de 33 semanas de edad gestacional a vitamina D 200 UI/kg día (máximo $400 \mathrm{UI} /$ día) o $960 \mathrm{UI} /$ día hasta los 3 meses de edad. A las 6 semanas de edad, la $25(\mathrm{OH}) \mathrm{D}$ fue significativamente mayor en el grupo de dosis altas. El único hallazgo significativo fue la ausencia de días de ventilación mecánica en el grupo de dosis altas, con una tendencia hacia la menor duración de los días de oxigenoterapia ${ }^{16}$. En el 2013, Ataseven y cols. realizaron un estudio de cohorte observacional evaluando la deficiencia de vitamina $\mathrm{D}$ como factor de riesgo para SDR. Se incluyeron un total de 129 recién nacidos de entre 29 y 35 semanas de edad gestacional. Todos tuvieron niveles de $25(\mathrm{OH}) \mathrm{D}$ menores de 75 $\mathrm{nmol} / \mathrm{L}$. No se encontró correlación entre edad gestacional y estatus de vitamina D. En un análisis no ajustado, el SDR se observó en un $28 \%$ del grupo con niveles menores a $25 \mathrm{nmol} / \mathrm{L}$ comparado con un $14 \%$ en aquellos con valores superiores, reduciendo la frecuencia de SDR en 3.34 veces en aquellos con los valores más altos ${ }^{17}$. Finalmente, en un estudio observacional, Koroglu y cols., encontraron un incremento promedio de 4 veces en el riesgo de desarrollar de DBP en comparación a aquellos sin este hallazgo ${ }^{18}$.

\section{Asma infantil y atopia}

Uno de los principales efectos asociados a la vitamina $\mathrm{D}$ es su propiedad inmunomoduladora. A nivel celular, la vitamina D modifica el patrón de respuesta inmune mediante la regulación del balance de citoquinas Th1/Th2, suprimiendo las Th1 (IL-2, IL-12, INF) e incrementando la producción de Th2 (IL-5, IL-10), además mantiene la homeostasis inmune a través de la supresión Th17 (IL-17) e inducción de células T reguladoras $\mathrm{CD} 4+\mathrm{CD} 25+(\text { tabla } 1)^{19}$. Adicionalmente, células inmunes como los macrófagos activados y células dendríticas han demostrado una producción local de $1,25(\mathrm{OH})_{2} \mathrm{D}$, la que es capaz de regular la expresión de proteínas antimicrobianas como las calecidinas y defensinas cuyo efecto restringe la respuesta inflamatoria $y$ atenúa la severidad de los fenotipos alérgicos ${ }^{20}$.

Una teoría emergente relaciona el impacto biológico del metabolismo de la vitamina $\mathrm{D}$ en el embarazo, basada en el rol inmunoprotector de la placenta tanto para la madre como para el feto. Variadas células placentarias capaces de expresar receptores Toll-like, tienen el potencial de responder a la infección e inducir la producción local de $1,25(\mathrm{OH})_{2} \mathrm{D}^{21}$.

Aún más, la deficiencia de vitamina $\mathrm{D}$ ha sido relacionada con cierto grupo de pacientes asmáticos resistentes a la acción de corticoides, mediante su acción directa sobre linfocitos y monocitos, favoreciendo la producción de la IL-10 antiinflamatoria inducida por esteroides. A su vez, su efecto inhibitorio sobre la proliferación del musculo liso en la vía aérea la postula como un potencial protector de la remodelación de la vía aérea en asmáticos ${ }^{22}$.

J Jat y cols. en un meta-análisis publicado el 2017, evaluaron la relación entre la ingesta prenatal de vitamina D y el desarrollo de enfermedades alérgicas en el hijo. Se incluyeron 3 estudios randomizados controlados por placebo (RCT), agrupando un total de 1493 niños. En relación con la presencia de sibilancias recurrentes a los 3 años de vida, los resultados mostraron una asociación entre la ingesta diaria de vitamina D (entre 800 a 4.000 UI) y una reducción de hasta un $33 \%$ en el desarrollo de sibilancias recurrentes ${ }^{23}$. Por otra parte, estudios observacionales en niños asmáticos han demostrado niveles de $25(\mathrm{OH}) \mathrm{D}$ entre 2 y 16 veces más bajos que aquellos no-asmáticos y un alto porcentaje de deficiencia e insuficiencia $(28,5$ y $26,7 \%$ respectivamente) de vitamina $D^{24}$.

Martineau y cols, en una revisión reciente de Cochrane, evaluaron la eficacia de la administración de vitamina $\mathrm{D}$ en la reducción del riesgo de desarrollar 
Tabla 1. Relación entre diversas patologías respiratorias pediátricas y Vitamina $D$

\begin{tabular}{|c|c|c|}
\hline Patología & Mecanismo propuesto & Manifestaciónes por déficit \\
\hline Asma & $\begin{array}{l}\text { Supresión de respuesta Th1 y potenciación de Th2 } \\
\text { Supresión de IL-17 } \\
\text { Inducción de células T reguladoras CD4+CD25+ } \\
\text { Inhibición de la proliferación del musculo liso bronquial }\end{array}$ & $\begin{array}{l}\text { Desarrollo precoz de enfermedad atópica } \\
\text { Incremento de exacerbaciones severas } \\
\text { Remodelación de la vía aérea }\end{array}$ \\
\hline Fibrosis quística & $\begin{array}{l}\text { Aumento de MAPK fosfatasa } 1 \text { y el factor nuclear kappa } \\
\text { B (NF-Kb) con actividad inhibitoria de los principales } \\
\text { factores pro-inflamatorios }\end{array}$ & $\begin{array}{l}\text { Bronquiectasias } \\
\text { Infecciones por Pseudomona } \\
\text { ¿Alteración de función pulmonar? }\end{array}$ \\
\hline Infecciones virales & $\begin{array}{l}\text { Modulación de IL-8 por medio de receptores TL3 } \\
\text { Expresión de péptidos antimicrobianos }\end{array}$ & Mayor susceptibilidad a infecciones respiratorias agudas \\
\hline Tuberculosis & $\begin{array}{l}\text { Efecto directo de catelicidinas sobre el } M \text {. Tuberculosis } \\
\text { Potencial función antimicrobiana de macrófagos media- } \\
\text { da por INF- } \gamma\end{array}$ & $\begin{array}{l}\text { ¿Alteración del periodo de conversión del esputo? } \\
\text { ¿Menor efecto inhibitorio del tratamiento? }\end{array}$ \\
\hline TRS & Desconocido & $\begin{array}{l}\text { Relación directa entre severidad del IAH y déficit de } \\
\text { vitamina D }\end{array}$ \\
\hline
\end{tabular}

TRS: Trastornos Respiratorios del Sueño, IAH: Indice Apnea-Hipopnea

Tabla 2. Vitamina D y patología respiratoria pediátrica: conclusiones de las principales revisiones sistemáticas y/o meta-análisis

\begin{tabular}{|c|c|c|}
\hline Patología & Principales resultados & Nivel de evidencia (GRADE) \\
\hline Asma & $\begin{array}{l}\text { Reducción de exacerbaciones severas en aquellos con déficit de vitamina D, sin } \\
\text { modificación de episodios leves }\end{array}$ & Evidencia de alta calidad \\
\hline Rinitis alérgica & $\begin{array}{l}\text { Menor sensibilización a aero-alérgenos en aquellos con niveles séricos de vitamina } D \\
\geq 75 \mathrm{nmol} / \mathrm{L} \text {, con tendencia a un menor riesgo de rinitis alérgica }\end{array}$ & No reportada \\
\hline Fibrosis quística & Sin evidencia clínica de beneficio & - \\
\hline Infecciones virales & Menor riesgo de infecciones respiratorias virales con suplementación diaria o semanal & Evidencia de alta calidad \\
\hline
\end{tabular}

exacerbaciones severas de asma y la mejoría en el control de síntomas; este meta-análisis incluyó 7 estudios, involucrando a un total de 435 niños y 658 adultos, la mayoría de ellos con asma moderada a severa. La administración de vitamina $\mathrm{D}$ redujo la tasa de exacerbaciones que requieren corticoides a razón de 71\% promedio, aunque para el análisis de este resultado en particular se incluyeron solo 22 pacientes pediátricos, desconociéndose además si este efecto estuvo o no limitado a aquellos con menores niveles plasmáticos basales de vitamina D. No se evidenció efecto significativo en la mejoría de la función pulmonar evaluada mediante el \% de Volumen Espiratorio Forzado en el primer segundo $\left(\mathrm{VEF}_{1}\right)$ predicho ni en la evaluación del control de asma ${ }^{25}$.

Con el objeto de confirmar los resultados publicados en la revisión Cochrane respecto al beneficio de la vitamina $\mathrm{D}$ en la reducción de exacerbaciones severas y su relación con sus niveles plasmáticos basales al momento de la suplementación; Jolliffe y cols, publicaron en la revista Lancet en octubre del 2017, una nueva revisión sistemática y meta-análisis que incluyó la información individual de cada participante. Se seleccionaron un total de 8 estudios y 978 participantes, de ellos 5 RCT fueron pediátricos con un total de 297 niños. Al excluir aquellos trabajos en los que se reportó $\leq 1$ exacerbación que requiriera corticoides para un meta-análisis final, de un total de 719 pacientes (150 pediátricos) la suplementación de vitamina $\mathrm{D}$ redujo entre un $8 \%$ y un $48 \%$ la tasa de exacerbaciones que requirieron tratamiento con corticoides sistémicos. En un análisis de un subgrupo de 92 pacientes categorizados según niveles basales de vitamina $\mathrm{D}$, el efecto protector de la suplementación fue relevante (hasta un $67 \%$ de reducción en exacerbaciones que requirieron corticoides) solo cuando los valores circulantes basales de $25(\mathrm{OH})$ D fueron $\leq 25 \mathrm{nmol} / \mathrm{L}(\text { tabla } 2)^{26}$.

\section{Fibrosis quística y bronquiectasias}

En la actualidad, el concepto que mejor explica la patogénesis de las bronquiectasias en niños es la "hipótesis del círculo vicioso". Este inicia con cualquier 
evento que altere el barrido mucociliar (infecciones del tracto respiratorio inferior, deshidratación del líquido periciliar y mucus) y permita el establecimiento de la colonización bacteriana en el tracto respiratorio inferior, predisponiendo a la formación de biofilm y la persistencia de una inflamación bronquial bacteriana, que conduce al daño de la superficie bronquial y finalmente al desarrollo de bronquiectasias ${ }^{27}$. Se ha teorizado que, dado las propiedades anti-infecciosas y anti-inflamatorias de la vitamina $\mathrm{D}$, esta puede jugar un rol en la patogénesis de la enfermedad.

Pese a la evidencia experimental, existen escasos estudios en humanos que evalúen el potencial efecto benéfico del incremento de los niveles plasmáticos de $25(\mathrm{OH})$ D sobre la inflamación pulmonar y las infecciones de la vía aérea en pacientes con Fibrosis Quística (FQ). Estudios retrospectivos en niños han encontrado correlación entre la severidad de la enfermedad pulmonar en FQ. Simoneau y cols. observaron que la insuficiencia de vitamina $\mathrm{D}$ estuvo asociada a infección bronquial por Pseudomona, así mismo, McCauley evidenció que los niveles de $25(\mathrm{OH})$ D estaban negativamente relacionados con la tasa de exacerbaciones pulmonares en niños y positivamente con el VEF1 en adolescentes ${ }^{28,29}$.

Ferguson y cols, en la revisión Cochrane más reciente, incluyeron por primera vez la evaluación de variables respiratorios en pacientes con FQ suplementados con vitamina D. No obstante, no se encontraron ensayos que reportaran control sobre la severidad de las bronquiectasias ni la proporción de aquellos que cursaron con exacerbaciones u hospitalizaciones. En cuanto a la función pulmonar, un único estudio en adultos mostró recuperación del $\mathrm{VEF}_{1}$ tras una caída del $10 \%$ o más al momento de la randomización, en el que $9 / 10$ pacientes lograron recuperar su función pulmonar basal comparado con $4 / 10$ en el grupo control $(\mathrm{P}=0,12)^{30}$.

\section{Infecciones respiratorias agudas}

Múltiples estudios in vitro han demostrado las funciones inmunomoduladoras de la vitamina $D$ en la inhibición de la proliferación celular, inducción de la diferenciación y apoptosis en el epitelio bronquial y macrófagos, junto con una disminución de la producción de citoquinas pro-inflamatorias ${ }^{31}$.

La $1,25(\mathrm{OH})_{2}$ D modula la expresión de IL-8 en las células del epitelio respiratorio mediada por receptores TLR3, reduce la expresión de marcadores patógenos Th17 y la subsecuente secreción de citoquinas proinflamatorias (IL-17A y INF- $\gamma$ ) así mismo, juega un rol crucial en la adecuada respuesta inmune local a virus incrementando la expresión en el epitelio respiratorio de péptidos antimicrobianos como la catelicidina y defensina $\beta 4$ en los macrófagos adyacentes y otras células inmunes innatas ${ }^{32,33}$.

Martineau y col., en una revisión sistemática y meta-análisis publicado el año 2017, utilizaron la información individualizada de cada participante, evaluando la relación entre la suplementación de vitamina $\mathrm{D}$ y la prevención de las infecciones respiratorias agudas. Se incluyó 25 estudios RCT para un total de 10.993 participantes, 6.650 de ellos $\leq$ a 16 años. En cuanto a la incidencia de infecciones respiratorias agudas, la suplementación de vitamina D se asoció a un efecto protector potente en aquellos con niveles plasmáticos de $25(\mathrm{OH}) \mathrm{D}$ menores a $25 \mathrm{nmol} / \mathrm{L}$, no así en aquellos con valores superiores. No obstante, al evaluar diferenciadamente según forma de administración, aquellos que recibieron suplementación diaria o semanal percibieron un beneficio aún mayor en la reducción de infecciones respiratorias, a razón de un 30\% promedio, al comparar con la suplementación en bolo. Dicho beneficio se extendió incluso a aquellos con niveles plasmáticos de vitamina $\mathrm{D}$ por sobre $25 \mathrm{nmol} / \mathrm{L}$. La administración en bolo no ofreció ningún beneficio incluso en aquellos con niveles plasmáticos bajos. Tanto la forma de administración como los niveles plasmáticos de vitamina $\mathrm{D}$ fueron 2 factores independientemente asociados a la incidencia de infecciones respiratorias $\operatorname{agudas}^{34}$.

\section{Bronquiolitis}

Diversas publicaciones han analizado la relación entre vitamina $\mathrm{D}$ e incidencia y/o severidad de bronquiolitis, con resultados contradictorios. En el 2014, Beigelman y cols. evaluaron la relación entre niveles plasmáticos de vitamina $\mathrm{D}$ al ingreso y la severidad del episodio agudo de bronquiolitis en lactantes menores de 1 año, considerando duración de la hospitalización, menor saturación de oxígeno y score de severidad (Score de Tal modificado). En una cohorte homogénea de 145 participantes, un 9,7\% presentó niveles plasmáticos $<20 \mathrm{ng} / \mathrm{mL}$, lo que se relacionó con menor edad y un menor consumo de fórmula láctea. Tras un ajuste de variables confundentes, no hubo diferencia en los indicadores de severidad entre aquellos con valores normales y deficiencia ${ }^{35}$.

Por el contrario, Moreno-Solis y cols. en un estudio transversal, analizaron una cohorte de 70 participantes entre 1 y 11 meses, encontrando niveles plasmáticos significativamente menores en aquellos con bronquiolitis moderada-severa versus controles sanos $(\mathrm{p}=0,022)$, con mayor prevalencia de insuficiencia/ deficiencia en aquellos más severos al compararlo con los casos leves, describiendo una correlación inversa 
significativa entre niveles plasmáticos de $25(\mathrm{OH})$ vitamina $\mathrm{D}$ y severidad ${ }^{36}$.

En el 2015, Saad y cols. publicaron el único RCT realizado a la fecha en el que se randomizó a 89 lactantes menores de 2 años a recibir vitamina D3 $100 \mathrm{UI} / \mathrm{Kg} /$ día por 7 días (en combinación a terapia estándar) versus placebo. Se evaluó duración de la hospitalización, tiempo de resolución de los síntomas, duración de la fluidoterapia intravenosa y duración de la oxígenoterapia. El grupo que recibió vitamina D tuvo una significativa menor duración de la hospitalización (139 vs 198 horas, $\mathrm{P}<0,00001)$, menor tiempo medio hasta la resolución de los síntomas (96 vs $145 \mathrm{~h}, \mathrm{P}<0,00001$ ) y un menor tiempo necesario para lograr la alimentación oral ( 20 vs 36 horas, $\mathrm{P}<0,00001$ ). No hubo diferencias en tiempo de resolución de la taquipnea, retracciones, duración de la fluidoterapia ni duración de la oxigenoterapia. No obstante, no se realizaron medición de valores basales de vitamina $\mathrm{D}$ ni posteriormente a los 7 días de su administración ${ }^{37}$.

\section{Tuberculosis}

La relación entre la Tuberculosis (TBC) y la vitamina D no es un hallazgo reciente. En la era pre antibiótica, el aceite de hígado de bacalao, una fuente primaria de vitamina $\mathrm{D}$, formaba un pilar importante en el tratamiento de esta enfermedad ${ }^{38}$. La deficiencia de vitamina $\mathrm{D}$ es un hecho frecuente en aquellos infectados con Tuberculosis, por lo que se cree que la depleción de vitamina $\mathrm{D}$ puede ser un predictor de TBC debido a su función esencial en el sistema inmune ${ }^{39}$. Se ha descrito un efecto antibacteriano directo de las catelicidinas sobre el Mycobacterium Tuberculosis, a su vez, la vitamina $D$ es esencial en la función antimicrobiana de los macrófagos mediada por INF- $\gamma$ y su efecto modulador sobre el fenotipo de las células $\mathrm{T}$ puede favorecer el control de la $\mathrm{TBC}^{40}$. Estudios en adultos han reportado múltiples beneficios, como por ejemplo un acortamiento en el periodo de conversión del esputo, mejoría en el efecto inhibitorio del tratamiento sobre el recuento de monocitos, citoquinas inflamatorias, quimioquinas y respuesta Th $1^{39}$. En niños, existe escasa información conocida que relacione los niveles séricos de vitamina $\mathrm{D}$ e inmunidad a TBC, sin embargo, un estudio reportó un $86 \%$ de deficiencia de vitamina $\mathrm{D}$ (menor a $20 \mathrm{nmol} / \mathrm{ml}$ ) o insuficiencia (menor a 75 $\mathrm{nmol} / \mathrm{ml}$ ) en niños con TBC activa ${ }^{41}$.

\section{Trastornos respiratorios del sueño}

Recientemente, se ha postulado la asociación entre el Síndrome de Apnea Hipopnea Obstructiva del Sueño (SAHOS) y menores niveles plasmáticos de vi- tamina $\mathrm{D}$, especialmente en obesos, existiendo una relación directa entre severidad de los índices respiratorios nocturnos e intensidad de la hipovitaminosis $\mathrm{D}^{42}$. Estudios preliminares sugieren que niños candidatos a adenotonsilectomía exhiben menores concentraciones séricas de $25(\mathrm{OH}) \mathrm{D}^{43}$.

En el 2014, Kheirandish-Gozal y cols. realizaron un estudio observacional y transversal en el que se reclutaron prospectivamente 176 niños derivados a un centro de estudio del sueño. Se les evaluó con polisomnografía nocturna y niveles plasmáticos de $25(\mathrm{OH}) \mathrm{D}$ a la mañana siguiente. Se dividió a los niños en 4 grupos según IMC y presencia de SAHOS, demostrando que aquellos niños obesos sin SAHOS expresaron niveles plasmáticos menores de vitamina $\mathrm{D}$ versus aquellos no-obesos sin SAHOS $(\mathrm{P}<0,01)$. De manera similar, aquellos no - obesos con SAHOS presentaron valores menores en comparación a controles no obesos $(\mathrm{P}<0,01)$. Aún más, al aplicar un modelo de regresión múltiple, se encontró una asociación lineal e independiente entre los niveles plasmáticos de vitamina $\mathrm{D}$ y los principales índices de polisomnográficos utilizados para caracterizar la severidad del SAHOS, el Índice de Apnea-Hipopnea (IAH) y el nadir de $\mathrm{SpO}_{2}{ }^{42}$.

\section{Conclusiones}

Las propiedades maduracionales de la vitamina $\mathrm{D}$ a nivel pulmonar, objetivadas in vitro, han sido pobremente evaluadas en la práctica, con estudios de moderada calidad que reportan una reducción en días de suplementación de oxígeno y un menor riesgo de SDR en prematuros.

Recientes meta-análisis, con evaluación individualizada de los participantes, muestran una significativa reducción en las exacerbaciones asmáticas severas e infecciones virales agudas (con suplementación diaria) en aquellos con bajos niveles plasmáticos de vitamina D. No obstante, la Global Initiative for Asthma (GINA) 2018 no la ubica en sus recomendaciones formales.

Nuevos estudios muestran un efecto terapéutico en aquellos hospitalizados por bronquiolitis severa, con evidencia contradictoria al relacionar niveles de insuficiencia/deficiencia con severidad. Pese a sus propiedades inmunomoduladoras, no se ha demostrado relación entre su suplementación y la evolución de la FQ y TBC pediátrica. Recientemente se ha explorado la asociación entre TRS y vitamina $\mathrm{D}$, demostrándose una relación directa entre severidad del IAH y déficit de vitamina D. No obstante, se desconocen su significancia a corto y largo plazo.

En consideración a los bajos costos de la suplementación de vitamina $\mathrm{D}$ y el gran problema económico que representan las exacerbaciones asmáticas e infecciones virales en niños, la suplementación de vitamina 
D representaría una estrategia potencialmente costoefectiva en la reducción de una importante causa de morbimortalidad pediátrica.

\section{Conflicto de intereses}

Los autores declaran no tener conflicto de intereses.

\section{Referencias}

1. Litonjua A. Childhood asthma may be a consequence of vitamin D deficiency. Curr Opin Allergy Clin Immunol. 2009; 9:202-7.

2. Janssens W, Bouillon R, Claes B, et al. Vitamin D deficiency is highly prevalent in COPD and correlates with variants in the vitamin D-binding gene. Thorax. 2010; 65:215-20.

3. Taher Y, van Esch B, Hofman G, Henricks P, van Oosterhout A. 1alpha,25dihydroxyvitamin D3 potentiates the beneficial effects of allergen immunotherapy in a mouse model of allergic asthma: role for IL-10 and TGFbeta. J Immunol. 2008; 180:5211-21.

4. Evans K, Nguyen L, Chan J, Innes B, Bulmer J, Kilby M, Hewison M. Effects of 25-hydroxyvitamin D3 and 1,25-dihydroxyvitamin D3 on cytokine production by human decidual cells. Biol Reprod. 2006;75:816-22.

5. Evans K, Bulmer J, Kilby M, Hewison M. Vitamin D and placental-decidual function. J Soc Gynecol Invest. 2004;11:263-71.

6. Lykkedegn S, Sorensen G, Beck-Nielsen $S$, Christesen $H$. The impact of vitamin D on fetal and neonatal lung maturation. A systematic review. Am J Physiol Lung Cell Mol Physiol. 2015;308:587-602.

7. Nguyen $M$, Trubert $C$, Rizk-Rabin M, Rehan V, Besancon F, Cayre Y, Garabedian M. 1,25-Dihydroxyvitamin D3 and fetal lung maturation: immunogold detection of VDR expression in pneumocytes type II cells and effect on fructose 1,6 bisphosphatase. J Steroid Biochem. 2004;89-90:93-7.

8. Le Roy, C, Reyes M, González J, PérezBravo F, Castillo-Duran C. Estado Nutricional de vitamina $\mathrm{D}$ en preescolares chilenos de zonas australes. Rev Med Chile. 2013; 141: 435-41.

9. Plum L, DeLuca H. Vitamin D, disease and terapeutic oportunities. Nat Rev Drug Discov. 2010;9:941-55.

10. Holick F. Vitamin D Deficiency. N Engl J Med. 2007; 357:266-81.

11. DeLuca H. Overview of general physiologic features and functions of vitamin D. Am J Clin Nutr. 2014; 80:1689-96.

12. Kaushal M, Magon N. Vitamin D in pregnancy: A metabolic outlook. Indian J Endocrinol Metab. 2013;17:76-82.

13. Sundar I, Hwang J, Wu S, Sun J, Rahman
I. Deletion of vitamin D receptor leads to premature emphysema/COPD by increased matrix metalloproteinases and lymphoid aggregates formation. Biochem Biophys Res Commun. 2011; 406: 127-33.

14. Lykkedegn S. The Impact of vitamin D on fetal and neonatal lung maduration. A systematic review. Am J Physiol Lung Cell Mol Physiol. 2015; 308:587-602.

15. Goyal R, Zhang L, Blood A, et al. Characterization of an animal model of pregnancyinduced vitamin D deficiency due to metabolic gene dysregulation. Am J Physiol Endocrinol Metab. 2014; 306: E256-66.

16. Backström M, Mäki R, Kuusela A, et al. Randomised controlled trial of vitamin D supplementation on bone density and biochemical indices in preterm infants. Arch Dis Child Fetal Neonatal. 1999; 80: F161-6.

17. Ataseven F, Aygün C, Okuyucu A, Bedir A, Kücük Y, Kücüködük S. Is Vitamin $D$ deficiency a risk factor for respiratory distress syndrome?. Int J Vitam Nutr. 2013; 83: 232-7.

18. Koroglu O, Onay H, Cakmak B, et al. Association of vitamin $\mathrm{D}$ receptor gene polymorphisms and bronchopulmonary dysplasia. Pediatr Res. 2014;76:171-6.

19. Papadopoulou A, Bountovi E, Papaevaggelou V, Priftis K. Maternal vitamin $\mathrm{D}$ status and development of asthma and allergy in early childhood. Mini Rev Med Chem. 2015; 15:900-12

20. Wang T, Nestel F, Bourdeau V, et al. Cutting edge: 1,25-dihydroxyvitamin $\mathrm{D} 3$ is a direct inducer of antimicrobial peptide gene expresión. J Immunol. 2004; 1;173:2909-12.

21. Vigano P, Mangioni S, Pompei F, Chiodo I. Maternal-Conceptus cross talk-a review. Placenta. 2003; 24: S56-61.

22. Pfeffer P, Hawrylowicz C. Vitamin D in asthma: Mechanisms of action and considerations for the clinical trials. Chest. 2017. pii: S0012-3692(17)32697-1.

23. Vahdaninia M, Mackenzie H, Helps S, Dean T. Prenatal intake of Vitamins and Allergic outcome in the offspring: A systematic review and meta-analysis. J Allergy Clin Immunol Pract. 2017;5:7718.

24. Jat K, Khairwa A. Vitamin D and asthma in children: A systematic review and meta-analysis of observational studies. Lung India. 2017; 34(4):355-63.

25. Martineau A, Cates C, Urashima M, et al.
Vitamin D for the managment of asthma. Cochrane Database Syst Rev. 2016; 9: CD011511.

26. Jolliffe D, Greenberg L, Hooper R, et al. Vitamin D supplementation to prevent asthma exacerbations: a systematic review and meta-analysis of individual participant data. Lancet Respir Med. 2017;5:881-90.

27. Moustaki M, Loukou I, Priftis K, Douros $\mathrm{K}$. Role of vitamin D in cystic fibrosis and non-cystic fibrosis bronchiectasis. World J Clin Pediatr. 2017;6:132-142.

28. Simoneau T, Bazzaz O, Sawicki G, Gordon C. Vitamin D status in children with cystic fibrosis. Associations with inflamation and bacterial colonization. Ann Am Thorac Soc. 2014;11:205-10.

29. McCauley L, Thomas W, Laguna T, Regelmann W, Moran A, Polgreen L. Vitamin D deficiency is associated with pulmonary exacerbations in children with cystic fibrosis. Ann Am Thorac Soc. 2014;11:198-204.

30. Ferguson J, Chang A. Vitamin D supplementation for cystic fibrosis. Cochrane Database Syst Rev. 2014;(5):CD007298.

31. Zdrenghea M, Makrinioti H, Bagacean C, Bush A, Johnston S, Stanciu L. Vitamin $D$ modulation of innate inmune response to respiratory viral infections. Rev Med Virol. 2017;27:1-12.

32. Hansdottir S, Monick M, Hinde S, Lovan N, Look D, Hunninghake G. Respiratory epitelial cells convert inactive vitamin $\mathrm{D}$ to its active form: potential effects on host defense. J Immunol. 2008;181:7090-9.

33. Liu P, Stenger S, Wenzel L, Tan B, Krutzik $S$, Ochoa M. Toll-Like receptor triggering of a vitamin D-mediated human antimicrobial response. Science. 2006; 311:1770-3.

34. Martineau R, Jolliffe D, Hooper R, Greenberg L, Aloia J, Bergman P, et al. Vitamin D supplementation to prevent acute respiratory tract infections: systematic review and meta-analysis of individual participant data. BMJ. 2017; 356:i6583.

35. Beigelman A, Castro M, Schwiger T, et al. Vitamin D leves are unrelated to the severity of respiratory syncytial virus bronchiolitis among hospitalized infantns. J Pediatric Infect Dis Soc. 2015;182-8.

36. Moreno G, Fernández F, Torres J, Torcello R, Gómez J, Perez J. Low serum 25-hydroxyvitamin D levels and 
bronchiolitis severity in spanish infants. Eur J Pediatr. 2015;365-72.

37. Saad K, Abd N, El-Houfey A, et al. Trial of vitamin $\mathrm{D}$ suplementation in infantis with bronchiolitis: A randomized, double -blind, placeb- controlled study. Pediatr Allergy Inmunol Pulmonol. 2015;26:102 - 105

38. Lalvani A, Connell D. Dissecting the immunological, antimicrobial and clinical effects of Vitamin D therapy in tuberculosis. Pathog Glob Health.
2012;106:378-9.

39. Hejazi M, Modarresi-Ghazani F, EntezariMaleki T. A review of vitamin D effects on common respiratory disease: Asthma, Chronic obstructive pulmonary disease, and tuberculosis. J Res Pharm Pract. 2016; 5:7-15.

40. Fabri M, Stenger S, Shin D, et al. Vitamin D is required for IFN-gammamediated antimicrobial activity of human macrophages. Sci Transl Med 2011;3(104):104ra102.
41. Williams B, Williams A, Anderson S. Vitamin D deficiency and insufficiency in children with tuberculosis. Pediatr Infect Dis J. 2008;27:941-2.

42. Kheirandish-Gozal L, Peris E, Gozal D. Vitamin D levels and obstructive sleep apnoea in children. Sleep Med. 2014; 15:459-63.

43. Reid D, Morton, Salkeld L, Bartley J. Vitamin D and tonsil diseasepreeliminary observations. Int J Pediatr Otorhinolaryngol. 2011; 75:261-4. 